\title{
Phase Angle from Bioelectric Impedance and Maturity-Related Factors in Adolescent Athletes: A Systematic Review
}

\author{
Aline Ferreira de Araújo Jerônimo ${ }^{1, *(\mathbb{D}}$, Nuno Batalha ${ }^{1,2}{ }^{\mathbb{D}}$, Daniel Collado-Mateo ${ }^{3}{ }^{(\mathbb{C})}$ and \\ Jose Alberto Parraca 1,2 (D) \\ 1 Departamento de Desporto e Saúde, Escola de Ciências e Tecnologia, Universidade de Évora, \\ Largo dos Colegiais 2, 7000-645 Évora, Portugal; nmpba@uevora.pt (N.B.); jparraca@uevora.pt (J.A.P.) \\ 2 Comprehensive Health Research Center (CHRC), University of Évora, Largo dos Colegiais 2, \\ 7000-645 Évora, Portugal \\ 3 Centre for Sport Studies, Rey Juan Carlos University, 28933 Madrid, Spain; danicolladom@gmail.com \\ * Correspondence: alinea.fje@gmail.com; Tel.: +351-935473635
}

Received: 22 April 2020; Accepted: 10 June 2020; Published: 12 June 2020

\begin{abstract}
Phase angle (PhA) is a body composition (BC) parameter from bioelectrical impedance analysis that is suggested as a marker of cell integrity and general health. In adolescent athletes, PhA values are used to monitor the effects of training and competitions and seem to vary according to age, having an upward trajectory during puberty. Since adolescence is a period composed of maturation stages, the aim of this systematic review was to investigate the relationship between PhA and maturation stage in adolescent athletes. A search was conducted in PubMed/MEDLINE, BVS, Web of Science (WOS), Scopus, Science Direct, and Academic Search Complete (EBSCO) databases up to May 2020. Eligibility criteria followed the PICOS strategy and studies with healthy athletes aged 10-19 years of any level of competition were included. Six cross-sectional studies and two longitudinal studies met the inclusion criteria. Results showed that early and mature athletes have higher PhA than those who are non-matured or maturated late. Thus, PhA is influenced by the pubertal status. Considering $B C$ is one of the factors influencing sports performance together with its growth-related changes, practitioners may use PhA values in BC analysis of adolescent athletes.
\end{abstract}

Keywords: phase angle; maturation; athlete; adolescent; bioelectrical impedance

\section{Introduction}

Body composition (BC) assessment in a sport context is considered as a relevant strategy to improve performance, as well as to discriminate athletes with different competitive levels, prevent injuries, and provide a body composition profile of athletes from different sports [1-3]. Regarding the $\mathrm{BC}$ evaluation in youth athletes, we must consider that adolescence is a stage of growth characterized by intense physiological changes in various body systems that may influence the physical capacities and athletic performance $[4,5]$.

Maturational status is one of the factors that may influence BC. Especially in athletes, sexual and somatic maturation has been studied to understand changes in fat mass percentage (FM\%) and free fat mass (FFM) [6,7]. Moreover, skeletal maturation status is also evaluated in young athletes and seems to be associated with FFM [8]. From the sports performance point of view, maturational status is also relevant, considering that young athletes who maturate earlier show greater velocity, endurance, and agility compared to their late mature peers, which increases the chances to be selected for elite teams $[9,10]$. This analysis is important because, without the maturation status, it will not 
be possible to compare and understand properly the adolescent athlete's performance, especially in different competitive levels [11].

Bioelectrical impedance analysis (BIA) is a non-invasive and easy-to-use method of assessing body composition that measures the opposition of body tissues to a low-level flow, changing an electric current radiofrequency [12]. In a healthy cell, when the electric current passes through the extra and intracellular fluids a low resistance (R) is offered, at the same time as the cell membrane offers a different and higher resistance represented by the reactance $\left(X_{c}\right)$ [13]. With $R$ and $X_{c}$ values, it is possible to obtain the phase angle $(\mathrm{PhA})$, which is calculated by the arctangent of $\left(\mathrm{X}_{\mathrm{c}} / \mathrm{R}\right) \times 180^{\circ} / \pi[14]$. The PhA is considered a clinically important parameter that would provide information about the health and integrity of cells, with high PhA associated with higher cellularity and better cell membrane or cell function and influenced by the level of physical activity [15].

The BIA method has been used in healthy children and $\mathrm{PhA}$ is associated with cardiorespiratory fitness and FFM, showing the importance of promoting health behavior related to exercise in childhood [16]. Therefore, PhA can be used to monitor the health and physical fitness of adolescents and it is indicated as a parameter of body composition that should be taken into account in the prescription of physical exercise [17]. Regarding PhA values in adolescent athletes, there are many observational studies that considered these data to monitor the short- and long-term changes induced by training and to provide reference values of body composition [18-20].

According to a systematic review published by Di Vincenzo et al. [21], PhA values vary according to age, and this parameter seems to be lower in young athletes than in adults. Besides the positive association of PhA with age until late adulthood, one cross-sectional study that reported data about the variability of $\mathrm{PhA}$ among adolescent athletes considering bone maturity was shown [22]. However, this finding does not clarify the relation between $\mathrm{PhA}$ and maturation, and no reference values are provided, probably due to the lack of studies at that time. In this context, as further studies have been published since then and considering the need to clarify the association between $\mathrm{PhA}$ and maturational status, the aim of this systematic review was to investigate the relationship between PhA from BIA and maturation stage in adolescent athletes. We hypothesized that the PhA will be increased when the maturational status is in early stages or the age was increased.

\section{Materials and Methods}

\subsection{Protocol}

This systematic review was performed in accordance with the Preferred Reporting Items for Systematic Reviews and Meta-Analyses (PRISMA) statement.

\subsection{Eligibility Criteria}

This study review was conducted in order to analyze the relation between PhA and status of maturation in the sport context. The PICOS strategy was defined, in which " $\mathrm{P}$ " corresponds to healthy athletes, with ages between 10 and 19 years old, of any ethnicity or sex. There was no intervention (" $\mathrm{I}$ ") to be evaluated. The comparison group ("C") was formed by athletes of different sport modalities or non-athletic individuals. The outcome " $\mathrm{O}$ " corresponded to PhA, and the study design "S" indicated observational approaches (cross-sectional or longitudinal).

Studies were included in this systematic review in accordance with the following inclusion criteria: (a) studies involving athletes of any level of competition, with or without a control group; (b) studies assessing maturational status; (c) full papers published in peer-reviewed and indexed journals; (d) studies evaluating PhA by BIA; (e) published in English. Studies that analyzed groups of adolescent athletes categorized by different age ranges were also included, assuming that individuals are probably at different stages of the maturation process. Besides that, studies on non-healthy athletes or athletes with injuries, duplicated papers, and reviews were excluded. 


\subsection{Information Sources and Search Strategy}

A search was conducted in May 2020. The electronic databases used were PubMed/MEDLINE, BVS, Web of Science (WOS), Scopus, Science Direct, and Academic Search Complete (EBSCO). The following terms and Boolean operators were used as search strategy: (BIVA (bioelectric impedance vector analysis) OR "phase angle") AND (adolescents OR children OR matur* OR puber*) AND (sport OR athletes OR players).

\subsection{Study Selection and Data Extraction}

Titles and abstracts from the studies on electronic databases were screened independently by two reviewers (AFAJ and JAP), and full text of all potentially relevant articles was investigated to guarantee the fit with eligibility criteria. The reference lists of selected articles were examined to search for other relevant investigations.

The following data were extracted from the selected studies: name of first author, year of publication, country of origin, aims of the investigation, study population (number of participants, gender, and age), type of sport, level of competition, type of study, instrument of BIA, maturation status assessment, age category, and main results.

\subsection{Risk of Bias}

To assess the methodological quality of studies, we used the Quality Assessment Tool for Observational Cohort and Cross-Sectional Studies published by the National Heart, Lung, and Blood Institute (https://www.nhlbi.nih.gov/health-topics/study-quality-assessment-tools). Previous reviews have used this tool to evaluate the risk of bias of cohort and cross-sectional studies [21,23]. The methodological approach included 14 criteria, which involve the research question, population definition, participation rate, recruitment, sample size, measures, and assessment, as well as outcomes and confounding variables.

Study quality was rated either good (low risk of bias), fair (moderate risk of bias), or poor (high risk of bias) as judged by two reviewers (AFAJ and JAP), and inconsistencies were resolved by consensus or by consulting a third researcher (DCM).

\section{Results}

The initial literature search in databases revealed a total of 87 articles, of which 42 were duplicate. After reading the title and the abstract, nine articles were excluded because they were not related to the topic and 13 because they were not performed in athletes. Fifteen studies were selected for full-text reading, of which six were excluded because they did not stratify the sample by different age ranges and another one because it was not written in English. Thus, eight studies met the inclusion criteria for the systematic review and were included in the qualitative analysis, as shown in Figure 1.

\subsection{Study Characteristics}

The leading characteristics of the selected studies are summarized in Tables 1 and 2. All the articles were written in English and published in the last six years. Regarding study locations, three were carried out in Brazil [19,22,24], four in Italy [20,25-27], and one in Spain [18]. From all the selected papers, six were cross-sectional and two were longitudinal studies.

In general, the studies assessed 1027 adolescent athletes between 9 and 18 years old, including more males $(n=813)$ than females $(n=214)$. Giorgi et al. [27] also analyzed a group of 79 elite male athletes between 18.2 and 24 years, which exceptionally was included in this review because it covered adolescents under 19 years old and served as a comparison group of younger adolescents. Most of the analyzed studies evaluated soccer players (37.5\%) at professional and elite levels. Koury et al. [19] also evaluated elite players, but from six different sports and recently, in the year of 2019, the same authors focused their attention on evaluating amateur players [24], as shown in Table 1. The other 
studies investigated athletes from artistic swimming (12.5\%), swimming (12.5\%), and road cycling $(12.5 \%)[18,20,27]$.

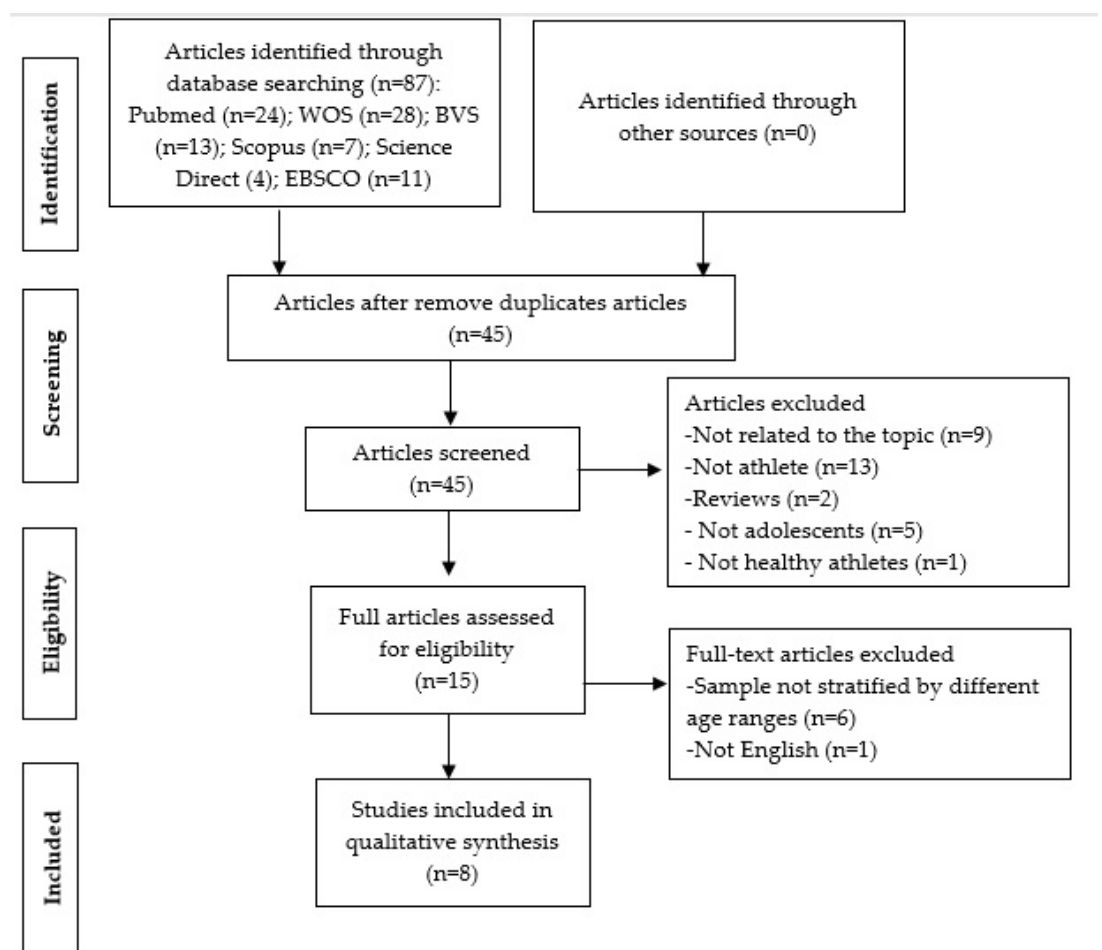

Figure 1. Flowchart of study selection in virtual databases.

\subsection{Maturation Status Assessment}

Skeletal maturation was evaluated in two studies and the authors used different protocols. The cross-sectional study by Koury et al. [22] used the Tanner-Whitehouse 3 method (TW3) that is based on X-ray measurements of 13 bones on the left hand. By this technique, it is possible to classify the individuals into early, on-time, and late maturity. The other study of Koury et al. [24] used dual-energy X-ray absorptiometry (DXA) images of hand and wrist to estimate bone age by the Greulich and Pyle method and classified the adolescents into mature or non-mature. Besides, they assessed sexual maturation status in females according to the age of menarche.

More recently, somatic maturation has gained interest. Campa et al. [25] and Toselli et al. [26] used an estimation of years from peak height velocity (PHV), which is an indicator for the adolescent growth spurt. The evaluation of somatic maturation status was done by Mirwald's equations and individuals are classified into early, on-time, or late maturation [28].

Regarding the participant's analysis, the sample of the selected articles was heterogeneous. Of 218 athletes that were classified according to the maturation status, $18 \%$ were in early-maturation, $66 \%$ on-time, and $35 \%$ presented late maturation [22,26]. Campa et al. [25] assessed somatic maturation but did not classify the athletes into groups, giving only the mean of years from PHV according to the age of individuals. Finally, Koury et al. [24] analyzed 318 (11-16 y) athletes and 31\% were categorized in the mature stage and $69 \%$ in the non-mature stage.

\subsection{Phase Angle Differences According to Maturation Stages}

\subsubsection{Skeletal Maturation}

Athletes classified according to skeletal maturation in the early category presented the highest values of PhA, as shown in Table 3. Koury et al. [22] evaluated forty male adolescent soccer players $(13.4 \pm 0.6 \mathrm{y})$ with a single-frequency tetrapolar analyzer. PhA values were higher in adolescents 
who maturated earlier compared to those considered on-time or who maturated late $(p=0.010)$. Furthermore, bone age and zinc concentration were responsible for $34 \%$ of $\mathrm{PhA}$ variability in the sample studied. Later, the same authors analyzed 167 male and 151 females athletes (11-16 y) and found that a higher PhA value was observed in mature adolescents for both sexes, indicating greater values of FFM $(p=0.002)$ [24], as shown in Table 3.

\subsubsection{Sexual Maturation}

Only Koury and colleagues [24] classified the female athletes according to sexual maturation. Following the same path, when athletes were classified by age of menarche in two categories (mature or immature), the mature adolescents presented PhA values significantly higher than immature ones $(p=0.001)$.

\subsubsection{Somatic Maturation}

Concerning years from PHV, Campa et al. [25] evaluated 249 male soccer players (9-19 y) and found that early somatic maturation was associated with accelerated development in $\mathrm{PhA}$ relative to players who maturated later $(p<0.05)$. Despite not categorizing the sample in groups according to maturation status, the authors showed that PhA values increase around - 1.5 years from PHV and keeps constant until advanced post-PHV, as shown in Table 3. Lastly, Toselli et al. [26] analyzed 178 male soccer players (12.1 $\pm 1.6 \mathrm{y})$ of first division using classic and specific bioelectric impedance vector analysis (BIVA). Although FFM values were higher in early-maturing athletes $(p<0.001)$, they did not find any association of PhA and somatic maturation status $(p=0.631)$, as shown in Table 3.

\subsection{Phase Angle Differences According to Chronological Age}

Four studies analyzed changes in PhA values according to age ranges of the adolescent athletes [18-20,27]. The cross-sectional study of Koury et al. [19] analyzed bioimpedance parameters from 105 individuals (12-19 years old) and shows a positive correlation between PhA and age. Besides that, athletes under 14 years old, who practiced football, had lower PhA values than athletic adolescents under 20 and basketball players under 18 years old, as shown in Table 4. Although these results have been attributed in part to biological maturation, it is important to highlight that factors related to training (intensity and/or volume) were pointed out as the main element of the equation.

Another cross-sectional study performed by Giorgi et al. [27] evaluated 59 youth elite (16.8 \pm 1.1 years) male road cyclists in comparison with others in different performance levels, including 79 elite athletes ( $21.1 \pm 2.9$ years). The PhA was higher in youth elite than in elite athletes, but no significant differences were reported between these groups, as shown in Table 4. Furthermore, the other bioimpedance parameters from the study indicated that youth athletes had equal soft tissue mass but to some extent higher body fluid volumes that could have been influenced by maturation level and body structure.

Longitudinal approaches also have been used to investigate changes in $\mathrm{PhA}$ in adolescent athletes. The study of Carrasco-Marginet et al. [18] showed an increase in PhA values after an artistic swimming training session in comen and junior swimmers. Regarding chronological age, a positive correlation with PhA was founded, so that junior athletes (16.3 \pm 0.6 years) had higher values of PhA than their comen $(13.9 \pm 0.9$ years) peers $(p=0.0001)$, as shown in Table 4 . In agreement with cross-sectional studies, Carrasco-Marginet et al. [18] highlight the need to investigate the influence of biological maturation in the relationship between PhA and chronological age. Finally, Meleleo et al. [20] followed for a year a sample of 29 athletes aged 8 to 11 years and an increase in PhA was observed but with no statistical significance $(p=0.91)$. 
Table 1. Description of selected studies by maturation status.

\begin{tabular}{|c|c|c|c|c|c|c|}
\hline Author/Year Publication & Country & Objectives & Sample & Sport/Level of Competition & Methods & $\begin{array}{l}\text { Maturation Status } \\
\text { Assessment }\end{array}$ \\
\hline Koury et al., 2018 [22] & Brazil & $\begin{array}{l}\text { To assess the associations between } \mathrm{PhA}{ }^{1} \\
\text { and body composition, skeletal maturity, } \\
\text { and zinc biochemical indices. }\end{array}$ & $\begin{array}{l}\mathrm{n}=40 \\
\text { Male } \\
\text { Age: } 13.4 \pm 0.6 \text { years }\end{array}$ & $\begin{array}{l}\text { Soccer } \\
\text { Professional }\end{array}$ & Cross-sectional & Skeletal maturation \\
\hline Koury et al., 2019 [24] & Brazil & $\begin{array}{l}\text { To evaluate the effect of biological } \\
\text { maturity on body composition in } \\
\text { Brazilian adolescent athletes. }\end{array}$ & $\begin{array}{l}\mathrm{n}=318 \\
\text { Males and Females } \\
\text { Age: } 11-16 \text { years }\end{array}$ & $\begin{array}{l}\text { Swimming, judo, badminton, } \\
\text { athletics, soccer, volleyball, } \\
\text { table tennis } \\
\text { Amateur }\end{array}$ & Cross-sectional & $\begin{array}{l}\text { Skeletal and sexual } \\
\text { maturation }\end{array}$ \\
\hline Campa et al., 2019 [25] & Italy & $\begin{array}{l}\text { To examine the influence of } \\
\text { chronological age and somatic } \\
\text { maturation on bioelectrical and body } \\
\text { composition parameters. }\end{array}$ & $\begin{array}{l}\mathrm{n}=249 \\
\text { Males } \\
\text { Age: } 9-17 \text { years }\end{array}$ & $\begin{array}{l}\text { Soccer } \\
\text { Elite }\end{array}$ & Cross-sectional & Somatic maturation \\
\hline Toselli et al., 2020 [26] & Italy & $\begin{array}{l}\text { To assess the influence of maturation on } \\
\text { body composition. }\end{array}$ & $\begin{array}{l}\mathrm{n}=178 \\
\text { Male } \\
\text { Age: } 12.1 \pm 1.6 \text { years }\end{array}$ & $\begin{array}{l}\text { Soccer } \\
\text { Elite }\end{array}$ & Cross-sectional & Somatic maturation \\
\hline
\end{tabular}


Table 2. Description of selected studies by chronological age.

\begin{tabular}{|c|c|c|c|c|c|c|}
\hline Author/Year Publication & Country & Objectives & Sample & Sport/Level of Competition & Methods & Results \\
\hline Koury et al., 2014 [19] & Brazil & $\begin{array}{l}\text { To assess } \mathrm{PhA}^{1} \text { and } \mathrm{BIVA}^{2} \text { in } \\
\text { adolescent and adult athletes practicing } \\
\text { a variety of sports. }\end{array}$ & $\begin{array}{l}\mathrm{n}=105 \\
\text { Males } \\
15.1 \pm 2.1 \text { years }\end{array}$ & $\begin{array}{l}\text { Athletics, Football, Swimming, } \\
\text { Water polo, Triathlon, } \\
\text { Basketball } \\
\text { Elite }\end{array}$ & Cross-sectional & $\begin{array}{l}\text { Adolescents had } \\
\text { lower PhA than } \\
\text { adults. }\end{array}$ \\
\hline $\begin{array}{l}\text { Carrasco-Marginet et al., } \\
2017 \text { [18] }\end{array}$ & Spain & $\begin{array}{l}\text { To determine the hydration changes } \\
\text { evoked during a training session. }\end{array}$ & $\begin{array}{l}\mathrm{n}=49 \\
\text { Females } \\
14.6 \pm 1.4 \text { years }\end{array}$ & Synchronized swimming & $\begin{array}{l}\text { Longitudinal } \\
\text { (Pre-post) }\end{array}$ & $\begin{array}{l}\text { PhA increases after a } \\
\text { training session and } \\
\text { was positively } \\
\text { correlated with } \\
\text { chronological age. }\end{array}$ \\
\hline Meleleo et al., 2017 [20] & Italy & $\begin{array}{l}\text { To evaluate lean and FM }{ }^{3} \text { in children } \\
\text { involved in daily competitive sports } \\
\text { when compared to sedentary controls. }\end{array}$ & $\begin{array}{l}\mathrm{n}=29 \text { (athletes) } \\
\text { mean age }=9.5 \text { years } \\
\mathrm{n}=190 \text { (non-athletes) } \\
\text { mean age }=9.3 \text { years } \\
\text { Males and females }\end{array}$ & $\begin{array}{l}\text { Swimming and gymnastics } \\
\text { Amateur }\end{array}$ & $\begin{array}{l}\text { Longitudinal } \\
\text { t0 } \\
\text { t1 (after } 6 \text { months) } \\
\text { t2 (after } 1 \text { year) }\end{array}$ & $\begin{array}{l}\text { PhA was lower in } \\
\text { competitive children } \\
\text { in } \mathrm{t} 0 \text { and } \mathrm{t} 1 .\end{array}$ \\
\hline Giorgi et al., 2018 [27] & Italy & $\begin{array}{l}\text { To establish reference bioimpedance } \\
\text { data for the road cycling population and } \\
\text { to compare various performance levels } \\
\text { of cyclists. }\end{array}$ & $\begin{array}{l}\mathrm{n}=138 \\
\text { G: youth elite } \\
16.6 \pm 1.1 \\
\text { G: elite } \\
21.1 \pm 2.9 \\
\text { Males }\end{array}$ & Road cyclists & Cross-sectional & $\begin{array}{l}\text { No significant } \\
\text { difference was related } \\
\text { between groups. }\end{array}$ \\
\hline
\end{tabular}

${ }^{1}$ Phase angle; ${ }^{2}$ bioelectric impedance vector analysis; ${ }^{3}$ fat mass. 
Table 3. Bioelectric impedance outcomes measure according to maturation stage.

\begin{tabular}{|c|c|c|c|c|c|c|}
\hline Authors & Instrument & Outcome Measure & Early Maturation $^{a}$ & On-Time Maturation $^{a}$ & Late maturation $^{\mathrm{a}}$ & Reported Results \\
\hline \multirow{6}{*}{ Koury et al. (2018) } & \multirow{5}{*}{$\begin{array}{l}\text { Quantum 101, RJL } \\
\text { System. }(800 \mu \mathrm{A}, 50 \mathrm{~Hz})\end{array}$} & $\mathrm{R}(\Omega)$ & $497.0 \pm 60.7$ & $528.3 \pm 61.2$ & $645.2 \pm 70.7$ & $\uparrow^{*}$ \\
\hline & & $\mathrm{Xc}_{\mathrm{c}}(\Omega)$ & $59.6 \pm 9.9$ & $59.9 \pm 9.4$ & $62.4 \pm 9.6$ & $=$ \\
\hline & & $\mathrm{R} / \mathrm{H}(\Omega / \mathrm{m})$ & $300.5 \pm 38.5$ & $321.6 \pm 37.0$ & $438.0 \pm 55.0$ & $\uparrow^{*}$ \\
\hline & & $\mathrm{X}_{\mathrm{c} / \mathrm{H}}(\Omega / \mathrm{m})$ & $36.1 \pm 6.8$ & $37.2 \pm 3.8$ & $43.7 \pm 5.3$ & $\uparrow^{*}$ \\
\hline & & $\operatorname{PhA}\left({ }^{\circ}\right)$ & $6.8 \pm 0.9$ & $6.5 \pm 0.6$ & $5.7 \pm 0.5$ & $\uparrow$ \\
\hline & & & Mature $(\mathrm{F} / \mathrm{M})^{\mathrm{a}}$ & & Immature $(\mathrm{F} / \mathrm{M})^{\mathrm{a}}$ & \\
\hline \multirow{13}{*}{ Koury et al. (2019) } & \multirow{12}{*}{$\begin{array}{l}\text { Bia } 450, \text { Biodynamics. } \\
(800 \mu \mathrm{A}, 50 \mathrm{~Hz})\end{array}$} & \multirow{7}{*}{$\begin{array}{l}\mathrm{R}(\Omega) \\
\mathrm{Xc}(\Omega) \\
\mathrm{R} / \mathrm{H}(\Omega / \mathrm{m}) \\
\mathrm{Xc} / \mathrm{H}(\Omega / \mathrm{m}) \\
\operatorname{PhA}\left({ }^{\circ}\right)\end{array}$} & $621.5 \pm 81.0 / 516.3 \pm 47.0$ & & $\begin{array}{l}669.2 \pm 96.3 / 586.6 \pm \\
83.8\end{array}$ & $\Delta$ \\
\hline & & & $69.9 \pm 10.2 / 61.7 \pm 7.5$ & & $69.0 \pm 11.6 / 63.7 \pm 9.6$ & $=$ \\
\hline & & & $388.8 \pm 50.5 / 301.3 \pm 32.7$ & & $432.8 \pm 32.0 / 373.6 \pm$ & $\Delta$ \\
\hline & & & $43.8 \pm 6.6 / 36.1 \pm 5.3$ & & 70.0 & $=/ \Delta$ \\
\hline & & & $6.5 \pm 0.9 / 6.8 \pm 0.6$ & & $44.5 \pm 7.9 / 40.5 \pm 7.6$ & $\Delta$ \\
\hline & & & & & $5.9 \pm 1.0 / 6.3 \pm 0.8$ & \\
\hline & & & Mature $(\mathrm{F} / \mathrm{M})^{\mathrm{b}}$ & & Immature $(\mathrm{F} / \mathrm{M})^{\mathrm{b}}$ & \\
\hline & & $\mathrm{R}(\Omega)$ & $634.9 \pm 87.6 / \mathrm{NR}$ & & $669.6 \pm 97.9 / \mathrm{NR}$ & $\Delta$ \\
\hline & & Xc $(\Omega)$ & $70.9 \pm 10.5 / \mathrm{NR}$ & & $65.9 \pm 10.9 / \mathrm{NR}$ & $\Delta$ \\
\hline & & $\mathrm{R} / \mathrm{H}(\Omega / \mathrm{m})$ & $397.9 \pm 55.4 / \mathrm{NR}$ & & $441.7 \pm 80.6 / \mathrm{NR}$ & $\Delta$ \\
\hline & & $\mathrm{X}_{\mathrm{c} / \mathrm{H}}(\Omega / \mathrm{m})$ & $44.5 \pm 6.9 / \mathrm{NR}$ & & $43.3 \pm 8.1 / \mathrm{NR}$ & $=$ \\
\hline & & $\operatorname{PhA}\left({ }^{\circ}\right)$ & $6.4 \pm 0.96 / \mathrm{NR}$ & & $5.6 \pm 0.95 / \mathrm{NR}$ & $\Delta$ \\
\hline & & & Early maturation ${ }^{\mathrm{c}}$ & On-time maturation ${ }^{c}$ & Late maturation ${ }^{\mathrm{c}}$ & \\
\hline \multirow{3}{*}{ Campa et al. (2019) } & \multirow{3}{*}{$\begin{array}{l}\text { BIA 101, Anniversary. } \\
(50 \mathrm{~Hz})\end{array}$} & $\mathrm{R} / \mathrm{H}(\Omega / \mathrm{m})$ & NR & NR & NR & NR \\
\hline & & $\mathrm{X}_{\mathrm{C} / \mathrm{H}}(\Omega / \mathrm{m})$ & NR & NR & NR & NR \\
\hline & & $\operatorname{PhA}\left({ }^{\circ}\right)$ & NR & NR & NR & $\uparrow$ \\
\hline \multirow{3}{*}{ Toselli et al. (2020) } & \multirow{3}{*}{$\begin{array}{l}\text { BIA 101, Anniversary. } \\
(50 \mathrm{~Hz})\end{array}$} & $\mathrm{R} / \mathrm{H}(\Omega / \mathrm{m})$ & $325.3 \pm 76.4$ & $387.4 \pm 77.7$ & $423.1 \pm 75.3$ & $\uparrow^{*}$ \\
\hline & & $\mathrm{X}_{\mathrm{C}} / \mathrm{H}(\Omega / \mathrm{m})$ & $35.7 \pm 6.1$ & $41.9 \pm 7.9$ & $45.1 \pm 6.2$ & $\uparrow^{*}$ \\
\hline & & $\operatorname{PhA}\left({ }^{\circ}\right)$ & $6.4 \pm 0.8$ & $6.3 \pm 0.9$ & $6.2 \pm 0.5$ & $=$ \\
\hline
\end{tabular}

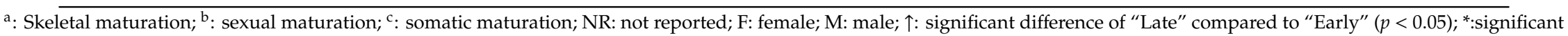
difference compared to "On-time" as the reference group $(p<0.05) ;=:$ no significant difference between groups; $\Delta$ : significant difference between groups $(p<0.05)$. 
Table 4. Bioelectrical impedance outcomes measure according to chronological age.

\begin{tabular}{|c|c|c|c|c|c|c|c|c|c|}
\hline Authors & Instrument & Outcome Measure & U20 & U18 & U16 & U14 & U12* & $\mathrm{U} 12+1 \mathrm{y} *$ & Reported Results \\
\hline \multirow{4}{*}{ Koury et al. (2014) } & \multirow{4}{*}{$\begin{array}{l}\text { Quantum 101, RJL } \\
\text { System } \\
(800 \mu \mathrm{A}, 50 \mathrm{~Hz})\end{array}$} & $\mathrm{R} / \mathrm{H}(\Omega / \mathrm{m})$ & $259.0 \pm 11.0^{\#}$ & $244.0 \pm 27.0^{£}$ & $238.0 \pm 28.0^{\beta}$ & $328.0 \pm 72.0^{\gamma}$ & - & - & $\Delta$ \\
\hline & & & $257.0 \pm 19.0 \S$ & $242.0 \pm 50.0^{\Omega}$ & & & & & \\
\hline & & $\mathrm{X}_{\mathrm{C}} / \mathrm{H}(\Omega / \mathrm{m})$ & $\begin{array}{l}37.6 \pm 3.6^{\#} \\
34.3 \pm 0.6 \$\end{array}$ & $\begin{array}{l}31.1 \pm 1.5^{£} \\
30.3 \pm 67^{\Omega}\end{array}$ & $33.5 \pm 3.5^{\beta}$ & $36.4 \pm 7.1^{\gamma}$ & - & - & $\Delta$ \\
\hline & & $\operatorname{PhA}\left(^{\circ}\right)$ & $\begin{array}{l}8.3 \pm 0.6^{\#} \\
7.6 \pm 0.4 \S\end{array}$ & $\begin{array}{l}7.1 \pm 0.8^{£} \\
7.2 \pm 0.8^{\Omega}\end{array}$ & $7.0^{\prime} \pm 0.5^{\beta}$ & $6.3 \pm 0.8^{\gamma}$ & - & - & $\Delta \downarrow$ \\
\hline \multirow{3}{*}{$\begin{array}{l}\text { Carrasco-Marginet et al. } \\
\text { (2017) }\end{array}$} & \multirow{3}{*}{$\begin{array}{l}\text { Z-Metrix, Bioparhom } \\
(74 \mu \mathrm{A}, 50 \mathrm{kHz})\end{array}$} & $\mathrm{R} / \mathrm{H}(\Omega / \mathrm{m})$ & - & $299.9 \pm 21.6$ & $328.4 \pm 38.8$ & - & - & - & $\Delta$ \\
\hline & & $\mathrm{Xc} / \mathrm{H}(\Omega / \mathrm{m})$ & - & $39.6 \pm 2.2$ & $40.0 \pm 4.5$ & - & - & - & $=$ \\
\hline & & $\operatorname{PhA}\left({ }^{\circ}\right)$ & - & $7.5 \pm 0.4$ & $7.0 \pm 0.5$ & - & - & - & $\Delta$ \\
\hline \multirow{4}{*}{ Meleleo et al., (2017) } & \multirow{4}{*}{$\begin{array}{l}\text { BIA 101, Akern, RJL } \\
\text { System } \\
(250 \mu \mathrm{A}, 50 \mathrm{~Hz})\end{array}$} & $\mathrm{R} / \mathrm{H}(\Omega / \mathrm{m})$ & - & - & - & - & $465.5 \pm 13.6^{\mathrm{a}}$ & $451.9 \pm 14.2^{\mathrm{a}}$ & $=$ \\
\hline & & $\mathrm{X}_{\mathrm{c} / \mathrm{H}}(\Omega / \mathrm{m})$ & - & - & - & - & $\begin{array}{l}418.7 \pm 14.9^{\mathrm{b}} \\
46.8 \pm 1.5^{\mathrm{a}}\end{array}$ & $\begin{array}{l}418.9 \pm 15.5^{\mathrm{b}} \\
47.1 \pm 2.0^{\mathrm{a}}\end{array}$ & $=$ \\
\hline & & & & & & & $40.5 \pm 1.6^{\mathrm{b}}$ & $42.6 \pm 2.2^{\mathrm{b}}$ & \\
\hline & & $\operatorname{PhA}\left({ }^{\circ}\right)$ & - & - & - & - & $\begin{array}{l}5.7 \pm 0.1 \mathrm{a} \\
5.5 \pm 0.1 \mathrm{~b}\end{array}$ & $\begin{array}{l}5.9 \pm 0.2^{\mathrm{a}} \\
5.8 \pm 0.2^{\mathrm{b}}\end{array}$ & $=$ \\
\hline \multirow{4}{*}{ Giorgi et al. (2018) } & \multirow{4}{*}{$\begin{array}{l}\text { BIA 101, Akern, RJL } \\
\text { System } \\
(250 \mu \mathrm{A}, 50 \mathrm{~Hz})\end{array}$} & $\mathrm{R} / \mathrm{H}(\Omega / \mathrm{m})$ & $284.5 \pm 31.4$ & $264.1 \pm 40.7$ & - & - & - & - & $\Delta$ \\
\hline & & & & & & & & & \\
\hline & & $\mathrm{X}_{\mathrm{C}} / \mathrm{H}(\Omega / \mathrm{m})$ & $34.9 \pm 4.1$ & $33.7 \pm 3.7$ & - & - & - & - & $=$ \\
\hline & & $\operatorname{PhA}\left({ }^{\circ}\right)$ & $7.0 \pm 0.7$ & $7.4 \pm 1.2$ & - & - & - & - & $=$ \\
\hline
\end{tabular}

\#: Athletics; §: triathlon; $€$ : water polo; $\Omega$ : basketball; $\beta$ : swimming; $\gamma$ : football; *: same group of individuals monitored longitudinally; a: female; b: male; =: no significant difference between groups; $\Delta$ : significant difference between groups $(p<0.05$ ); $\downarrow$ : significant difference between U14 compared to U18 (basketball) and U20 (athletics). 


\subsection{Risk of Bias}

The cross-sectional studies analyzed in the present review were considered as having a moderate risk of bias, which is mostly caused by the methodological design. In relation to longitudinal studies, no justification was provided to explain the sample size, which was, in general, small. Despite that, the two longitudinal studies included in this review had a moderate risk of bias, as shown in Table 5 .

Table 5. Quality Assessment Tool for Observational Cohort and Cross-Sectional Studies.

\begin{tabular}{|c|c|c|c|c|c|c|c|c|}
\hline \multirow[b]{2}{*}{ Criteria } & \multicolumn{4}{|c|}{ Maturation } & \multicolumn{4}{|c|}{ Age } \\
\hline & $\begin{array}{l}\text { Koury } \\
2018\end{array}$ & $\begin{array}{l}\text { Koury } \\
2019\end{array}$ & $\begin{array}{c}\text { Campa } \\
2019\end{array}$ & $\begin{array}{c}\text { Toselli } \\
2020\end{array}$ & $\begin{array}{l}\text { Koury } \\
2014\end{array}$ & $\begin{array}{l}\text { Carrasco- } \\
\text { Marginet } \\
2017\end{array}$ & $\begin{array}{c}\text { Meleleo } \\
2017\end{array}$ & $\begin{array}{l}\text { Giorgi } \\
2018\end{array}$ \\
\hline $\begin{array}{l}\text { 1. Was the research question or objective in this } \\
\text { paper clearly stated? }\end{array}$ & Yes & Yes & Yes & Yes & Yes & Yes & Yes & Yes \\
\hline $\begin{array}{l}\text { 2. Was the study population clearly specified } \\
\text { and defined? }\end{array}$ & Yes & Yes & Yes & Yes & Yes & Yes & Yes & Yes \\
\hline $\begin{array}{l}\text { 3. Was the participation rate of eligible persons } \\
\text { at least } 50 \% \text { ? }\end{array}$ & NR & NR & NR & NR & NR & Yes & NR & NR \\
\hline $\begin{array}{l}\text { 4. Were all the subjects selected or recruited from } \\
\text { the same or similar populations (including the same } \\
\text { time period)? Were inclusion and exclusion criteria } \\
\text { for being in the study prespecified and applied } \\
\text { uniformly to all participants? }\end{array}$ & Yes & Yes & Yes & Yes & Yes & Yes & No & No \\
\hline $\begin{array}{l}\text { 5. Was a sample size justification, power description, } \\
\text { or variance and effect estimates provided? }\end{array}$ & No & No & No & No & No & No & No & No \\
\hline $\begin{array}{l}\text { 6. For the analyses in this paper, were the } \\
\text { exposure(s) of interest measured prior to the } \\
\text { outcome(s) being measured? }\end{array}$ & No & No & No & No & No & Yes & Yes & No \\
\hline $\begin{array}{l}\text { 7. Was the timeframe sufficient so that one could } \\
\text { reasonably expect to see an association between } \\
\text { exposure and outcome if it existed? }\end{array}$ & No & No & No & No & No & Yes & Yes & No \\
\hline $\begin{array}{l}\text { 8. For exposures that can vary in amount or level, } \\
\text { did the study examine different levels of the } \\
\text { exposure as related to the outcome (e.g., categories } \\
\text { of exposure, or exposure measured as } \\
\text { continuous variable)? }\end{array}$ & NA & NA & NA & NA & NA & NA & NA & Yes \\
\hline $\begin{array}{l}\text { 9. Were the exposure measures (independent } \\
\text { variables) clearly defined, valid, reliable, and } \\
\text { implemented consistently across all study } \\
\text { participants? }\end{array}$ & Yes & No & Yes & Yes & Yes & Yes & Yes & Yes \\
\hline $\begin{array}{l}\text { 10. Was the exposure(s) assessed more than once } \\
\text { over time? }\end{array}$ & No & No & No & No & No & Yes & Yes & No \\
\hline $\begin{array}{l}\text { 11. Were the outcome measures (dependent } \\
\text { variables) clearly defined, valid, reliable, and } \\
\text { implemented consistently across all } \\
\text { study participants? }\end{array}$ & Yes & Yes & Yes & Yes & Yes & Yes & Yes & Yes \\
\hline $\begin{array}{l}\text { 12. Were the outcome assessors blinded to the } \\
\text { exposure status of participants? }\end{array}$ & NR & NR & NR & NR & NR & NR & NR & NR \\
\hline 13. Was loss to follow-up after baseline $20 \%$ or less? & NA & NA & NA & NA & NA & NA & Yes & NA \\
\hline $\begin{array}{l}\text { 14. Were key potential confounding variables } \\
\text { measured and adjusted statistically for their impact } \\
\text { on the relationship between exposure(s) } \\
\text { and outcome(s)? }\end{array}$ & Yes & NA & NA & NA & Yes & NA & Yes & No \\
\hline
\end{tabular}

Legend: NR (not reported); NA (not applicable).

\section{Discussion}

The purpose of this systematic review was to show the association of PhA from BIA and maturational status in adolescent athletes. Results showed that only four studies assessed PhA making an association with pubertal status, while another four papers considered chronological age. Considering all the three different methods of assessing maturation, early and mature athletes have higher PhA than those who are non-mature or maturated late. Regarding age, between youth athletes there is a positive correlation with $\mathrm{PhA}$, with biological maturation being the factor that could explain this relation.

In general, PhA has a stronger association with muscle performance, being considered as a health marker, especially in young people [29]. While high PhA values are related to FFM, cardiorespiratory fitness, and muscle strength [16,30], low PhA could indicate muscle injuries [31], malnutrition [32], or fatigue [30]. Physical exercise appears as a modifier agent of $\mathrm{PhA}$, as suggested in a recent meta-analysis 
performed by Mundstock et al. [15], who concluded that physical training, especially resistance training which increases muscle mass, had a positive effect on PhA. This reinforces the use of PhA values by researchers and practitioners to monitor the athlete's general health status, as well as an indicator of performance and muscle quality.

In sport, several authors investigated PhA values in adolescent athletes to analyze the effects of training, but mostly in swimming $[18,20,33]$. In these studies, changes in PhA varied according to chronological age and level of competition. Indeed, in adolescents, it is known that PhA increases with age, unlike what occurs in adults [21]. On the other hand, individuals with the same chronological age can be in different maturation statuses [34]. Considering that early maturated athletes demonstrate greater strength, speed, and agility compared to those who maturated late [35,36], sports professionals and researchers may need further information to adequately evaluate the performance of young and adolescent athletes.

Reference values of PhA are well known for non-athletic adolescents [29,37,38]. However, even in the non-athletic population, no reference PhA values take into account maturation status. Only a few number of studies discussed this relation. Although some authors indicated that body composition variables such as PhA are independent of maturation status in adolescents [39,40], another study pointed out that this parameter is higher in individuals who matured early, differing between sex [41].

In this review, we also revealed the assessment methods of maturational status used in the studies about PhA from BIA in adolescent athletes. Skeletal and somatic maturation were the most used among authors, including X-ray images and equations to predict PHV, respectively. Both methods are well established in the literature as being accurate in assessing maturation status [42-44]. Furthermore, as we could see in our review, the PhA and maturation stage relationship seems not to be influenced by the method of assessment of pubertal status, since different approaches showed a positive relationship between the variables. Despite that, further studies in this area are necessary to investigate whether the type of maturation assessment can influence PhA analysis in adolescent athletes. In practical terms, assessing skeletal maturation could represent a limitation due to the need for radiological methods that are more expensive.

Therefore, since adolescence is a critical period that requires substantial knowledge about $\mathrm{BC}$, the present review contributes with a better understanding of the importance of considering the maturation stage in the analysis of young athletes' $\mathrm{PhA}$ value. Moreover, understanding changes in physiological adaptations associated with a growth spurt becomes relevant in terms of training prescription and injury prevention [45]. We suggest that PhA values should be used in BC analysis of adolescent athletes and that pubertal status should also be considered. For future investigations, the maturation stage must be classified more homogeneously, so that reference values can be drawn for each stage.

The current study presents slight limitations that must be mentioned. Only studies written in English and indexed in the selected scientific databases were included. Results showed that only four studies assessed PhA making an association with pubertal status, while another four papers considered chronological age; therefore, we consider that more studies are needed, mainly of a longitudinal character. Furthermore, due to the heterogeneity in the ways of classifying the maturation stage, it was not possible to establish reference values for the study population. Another potential limitation could be related to the absence of registration in PROSPERO. Finally, the risk of bias was moderate in most parts of the studies, due to the cross-sectional design. Despite these three limitations, this review provides relevant information about the relation between $\mathrm{PhA}$ and maturation status in young and adolescent athletes.

\section{Conclusions}

As the PhA is related to the maturational state, it should be considered in the assessment of $\mathrm{BC}$ in young and adolescent athletes. This association is observed regardless of the method used to evaluate the maturational stage. Therefore, researchers, coaches, and practitioners can use PhA to monitor $\mathrm{BC}$ parameters considering the pubertal status, especially in training prescription and performance 
assessment. However, given the low number of studies and the risk of bias observed, there is a need to investigate the relation between pubertal stage and $\mathrm{PhA}$ and to what extent this BIA parameter varies in each maturation stage in a healthy athletic population. In this regard, longitudinal studies are especially recommended to understand this relationship over time.

Author Contributions: Conceptualization, A.F.d.A.J., J.A.P., and N.B.; methodology, A.F.d.A.J. and J.A.P.; validation, N.B. and J.A.P.; formal analysis, A.F.d.A.J., J.A.P., and D.C.-M.; investigation, A.F.d.A.J.; resources, A.F.d.A.J.; data curation, A.F.d.A.J., J.A.P., and D.C.-M.; writing-original draft preparation, A.F.d.A.J.; writing - review and editing, J.A.P., D.C.-M., and N.B.; supervision, J.A.P.; project administration, N.B. and J.A.P.; funding acquisition, J.A.P. All authors have read and agree to the published version of the manuscript.

Funding: This research was funded by the Instituto Português do Desporto e Juventude UÉvora-UniverCIDADE V program CP /696/DDT/2019).

Conflicts of Interest: The authors declare no conflict of interest. The funders had no role in the design of the study; in the collection, analyses, or interpretation of data; in the writing of the manuscript, or in the decision to publish the results.

\section{References}

1. Barbieri, D.; Zaccagni, L.; Babić, V.; Rakovac, M.; Mišigoj-Duraković, M.; Gualdi-Russo, E. Body composition and size in sprint athletes. J. Sports Med. Phys. Fitness 2017, 57, 1142-1146. [CrossRef] [PubMed]

2. Campa, F.; Matias, C.; Gatterer, H.; Toselli, S.; Koury, J.C.; Andreoli, A.; Melchiorri, G.; Sardinha, L.B.; Silva, A.M. Classic Bioelectrical Impedance Vector Reference Values for Assessing Body Composition in Male and Female Athletes. Int. J. Environ. Res. Public Health 2019, 16, 5066. [CrossRef] [PubMed]

3. Silva, A.M. Structural and functional body components in athletic health and performance phenotypes. Eur. J. Clin. Nutr. 2019, 73, 215-224. [CrossRef] [PubMed]

4. Naughton, G.; Farpour-Lambert, N.J.; Carlson, J.; Bradney, M.; Van Praagh, E. Physiological issues surrounding the performance of adolescent athletes. Sport. Med. 2000, 30, 309-325. [CrossRef] [PubMed]

5. Pinto, V.C.M.; dos Santos, P.G.M.D.; Medeiros, R.C.D.S.; Souza, F.E.S.; Simões, T.B.D.S.; Dantas, R.P.N.D.C.; Cabral, B.G.D.A.T. Estágios maturacionais: Comparação de indicadores de crescimento e capacidade física em adolescentes. J. Hum. Growth Dev. 2018, 28, 42-49. [CrossRef]

6. Luiz-de-Marco, R.; Gobbo, L.A.; Castoldi, R.C.; Maillane-Vanegas, S.; da Silva Ventura Faustino-da-Silva, Y.; Exupério, I.N.; Agostinete, R.R.; Fernandes, R.A. Impact of changes in fat mass and lean soft tissue on bone mineral density accrual in adolescents engaged in different sports: ABCD Growth Study. Arch. Osteoporos. 2020, 15, 1-8. [CrossRef]

7. Malina, R.M. Body Composition in Athletes: Assessment and Estimated Fatness. Clin. Sports Med. 2007, 26, 37-68. [CrossRef]

8. Teixeira, A.S.; Fernandes, J.; Cristina, P.; Cesar, P.; Campos, F.D.S.; Lucas, R.D.D.; Guilherme, L.; Guglielmo, A. Relative age effect, skeletal maturation and aerobic running performance in youth soccer players. Motriz 2018, 24, 1-8. [CrossRef]

9. Rommers, N.; Mostaert, M.; Goossens, L.; Vaeyens, R.; Witvrouw, E.; Lenoir, M.; D'Hondt, E. Age and maturity related differences in motor coordination among male elite youth soccer players. J. Sports Sci. 2018, 37, 196-203. [CrossRef]

10. Torres-Unda, J.; Zarrazquin, I.; Gil, J.; Ruiz, F.; Irazusta, A.; Kortajarena, M.; Seco, J.; Irazusta, J. Anthropometric, physiological and maturational characteristics in selected elite and non-elite male adolescent basketball players. J. Sports Sci. 2013, 31, 196-203. [CrossRef]

11. Trecroci, A.; Milanović, Z.; Frontini, M.; Iaia, F.M.; Alberti, G. Physical Performance Comparison between under 15 Elite and Sub-Elite Soccer Players. J. Hum. Kinet. 2018, 61, 209-216. [CrossRef] [PubMed]

12. Castizo-Olier, J.; Irurtia, A.; Jemni, M.; Carrasco-Marginet, M.; Fernández-García, R.; Rodríguez, F.A. Bioelectrical impedance vector analysis (BIVA) in sport and exercise: Systematic review and future perspectives. PLoS ONE 2018, 13, e0197957. [CrossRef] [PubMed]

13. Fornazari, E.; Los, N.A.; Luiz, S., Jr. Bioimpedância: Introdução e utilizações de técnicas de bioimpedância. In Proceedings of the SEA-Seminário de Eletrônica e Automação, Paraná, Brazil., 25 September 2017; pp. 1-5.

14. Kumar, S.; Dutt, A.; Hemraj, S.; Bhat, S.; Manipadybhima, B. Phase Angle Measurement in Healthy Human Subjects through Bio-Impedance Analysis. Iran. J. Basic Med. Sci. 2012, 15, 1180-1184. [PubMed] 
15. Mundstock, E.; Amaral, M.A.; Baptista, R.R.; Sarria, E.E.; dos Santos, R.R.G.; Filho, A.D.; Rodrigues, C.A.S.; Forte, G.C.; Castro, L.; Padoin, A.V.; et al. Association between phase angle from bioelectrical impedance analysis and level of physical activity: Systematic review and meta-analysis. Clin. Nutr. 2019, 38, 1504-1510. [CrossRef]

16. Langer, R.D.; da Costa, K.G.; Bortolotti, H.; Fernandes, G.A.; de Jesus, R.S.; Gonçalves, E.M. Phase angle is associated with cardiorespiratory fitness and body composition in children aged between 9 and 11 years. Physiol. Behav. 2020, 215, 112772. [CrossRef]

17. Martins, P.C.; de Lima, L.R.A.; Berria, J.; Petroski, E.L.; Silva, A.M.; Silva, D.A.S. Association between phase angle and isolated and grouped physical fitness indicators in adolescents. Physiol. Behav. 2020, 217, 112825. [CrossRef]

18. Carrasco-Marginet, M.; Castizo-Olier, J.; Rodríguez-Zamora, L.; Iglesias, X.; Rodríguez, F.A.; Chaverri, D.; Brotons, D.; Irurtia, A. Bioelectrical impedance vector analysis (BIVA) for measuring the hydration status in young elite synchronized swimmers. PLoS ONE 2017, 12, e0178819. [CrossRef]

19. Koury, J.C.; Trugo, N.M.F.; Torres, A.G. Phase angle and bioelectrical impedance vectors in adolescent and adult male athletes. Int. J. Sports Physiol. Perform. 2014, 9, 798-804. [CrossRef]

20. Meleleo, D.; Bartolomeo, N.; Cassano, L.; Nitti, A.; Susca, G.; Mastrototaro, G.; Armenise, U.; Zito, A.; Devito, F.; Scicchitano, P.; et al. Evaluation of body composition with bioimpedence. A comparison between athletic and non-athletic children. Eur. J. Sport Sci. 2017, 17, 710-719. [CrossRef]

21. Di Vincenzo, O.; Marra, M.; Scalfi, L. Bioelectrical impedance phase angle in sport: A systematic review. J. Int. Soc. Sports Nutr. 2019, 16,1-11. [CrossRef]

22. Koury, J.C.; de Oliveira-Junior, A.V.; Portugal, M.R.C.; de Oliveira, K.D.J.F.; Donangelo, C.M. Bioimpedance parameters in adolescent athletes in relation to bone maturity and biochemical zinc indices. J. Trace Elem. Med. Biol. 2018, 46, 26-31. [CrossRef] [PubMed]

23. Diez-Fernández, A.; Álvarez-Bueno, C.; Martínez-Vizcaíno, V.; Sotos-Prieto, M.; Recio-Rodríguez, J.I.; Cavero-Redondo, I. Total dairy, cheese and milk intake and arterial stiffness: A systematic review and meta-analysis of cross-sectional studies. Nutrients 2019, 11, 741. [CrossRef] [PubMed]

24. Koury, J.C.; Ribeiro, M.A.; Massarani, F.A.; Vieira, F.; Marini, E. Fat-free mass in adolescent athletes: Accuracy of bioimpedance equations and identification of new predictive equations. Nutrition 2019, 60, 59-65. [CrossRef] [PubMed]

25. Campa, F.; Silva, A.M.; Iannuzzi, V.; Mascherini, G.; Benedetti, L.; Toselli, S. The Role of Somatic Maturation on Bioimpedance Patterns and Body Composition in Male Elite Youth Soccer Players. Int. J. Environ. Res. Public Health 2019, 16, 4711. [CrossRef]

26. Toselli, S.; Marini, E.; Latessa, P.M.; Benedetti, L.; Campa, F. Maturity Related Di ff erences in Body Composition Assessed by Classic and Specific Bioimpedance Vector Analysis among Male Elite Youth Soccer Players. Int. J. Environ. Res. Public Health 2020, 17, 729. [CrossRef]

27. Giorgi, A.; Vicini, M.; Pollastri, L.; Lombardi, E.; Magni, E.; Andreazzoli, A.; Orsini, M.; Bonifazi, M.; Lukaski, H.; Gatterer, H. Bioimpedance patterns and bioelectrical impedance vector analysis (BIVA) of road cyclists. J. Sports Sci. 2018, 36, 2608-2613. [CrossRef]

28. Mirwald, R.L.; Baxter-Jones, A.D.G.; Bailey, D.A.; Beunen, G.P. An assessment of maturity from anthropometric measurements. Med. Sci. Sports Exerc. 2002, 34, 689-694. [CrossRef] [PubMed]

29. Hetherington-Rauth, M.; Baptista, F.; Sardinha, L.B. BIA-assessed cellular hydration and muscle performance in youth, adults, and older adults. Clin. Nutr. 2019. [CrossRef]

30. Nunes, J.P.; Ribeiro, A.S.; Silva, A.M.; Schoenfeld, B.J.; Dos Santos, L.; Cunha, P.M.; Nascimento, M.A.; Tomeleri, C.M.; Nabuco, H.C.G.; Antunes, M.; et al. Improvements in phase angle are related with muscle quality index after resistance training in older women. J. Aging Phys. Act. 2019, 27, 515-520. [CrossRef]

31. Nescolarde, L.; Yanguas, J.; Terricabras, J.; Lukaski, H.; Alomar, X.; Rosell-Ferrer, J.; Rodas, G. Detection of muscle gap by L-BIA in muscle injuries: Clinical prognosis. Physiol. Meas. 2017, 38, L1-L9. [CrossRef]

32. Player, E.L.; Morris, P.; Thomas, T.; Chan, W.Y.; Vyas, R.; Dutton, J.; Tang, J.; Alexandre, L.; Forbes, A. Bioelectrical impedance analysis (BIA)-derived phase angle (PA) is a practical aid to nutritional assessment in hospital in-patients. Clin. Nutr. 2019, 38, 1700-1706. [CrossRef] 
33. Irurtia, A.; Carrasco-Marginet, M.; Rodríguez-Zamora, L.; Iglesias, X.; Brontos, D.; Rodriguez, F. Bioelectrical impedance vector migration induced by training in young competitive synchronized swimmers. In XIIth International Symposium for Biomechanics and Medicine in Swimming; Australian Institute of Sport: Canberra, Australian, 2014; pp. 426-430. [CrossRef]

34. Milani, S.; Benso, L. Why we can't determine reliably the age of a subject on the basis of his maturation degree. J. Forensic Leg. Med. 2019, 61, 97-101. [CrossRef] [PubMed]

35. Guimarães, E.; Ramos, A.; Janeira, M.A.; Baxter-Jones, A.D.G.; Maia, J. How Does Biological Maturation and Training Experience Impact the Physical and Technical Performance of 11-14-Year-Old Male Basketball Players? Sports 2019, 7, 243. [CrossRef] [PubMed]

36. Meyers, R.W.; Oliver, J.L.; Hughes, M.G.; Lloyd, R.S.; Cronin, J.B. The Influence of Maturation on Sprint Performance in Boys over a 21-Month Period. Med. Sci. Sports Exerc. 2016, 48, 2555-2562. [CrossRef] [PubMed]

37. Anja, B.W.; Danielzik, S.; Dörhöfer, R.P.; Later, W.; Wiese, S.; Müller, M.J. Phase angle from bioelectrical impedance analysis: Population reference values by age, sex, and body mass index. J. Parenter. Enter. Nutr. 2006, 30, 309-316. [CrossRef]

38. Kuchnia, A.J.; Teigen, L.M.; Cole, A.J.; Mulasi, U.; Gonzalez, M.C.; Heymsfield, S.B.; Vock, D.M.; Earthman, C.P. Phase Angle and Impedance Ratio: Reference Cut-Points From the United States National Health and Nutrition Examination Survey 1999-2004 From Bioimpedance Spectroscopy Data. J. Parenter. Enter. Nutr. 2017, 41, 1310-1315. [CrossRef]

39. Cumpian-Silva, J.; Rinaldi, A.E.M.; Mazzeti, C.M.D.S.; Conde, W.L. Body phenotypes in adolescence and sexual maturation. Cad. Saude Publica 2018, 34, e00057217. [CrossRef]

40. Oliveira, J.R. De Maturação Sexual e Adiposidade em Crianças e Adolescentes de duas Escolas de São Paulo. Doctoral Dissertation, Universidade de São Paulo, São Paulo, Brazil, 2010.

41. Buffa, R.; Floris, G.; Marini, E. Bioelectrical impedance vector in pre- and postmenarcheal females. Nutrition 2002, 18, 474-478. [CrossRef]

42. Walker, I.V.; Smith, C.R.; Davies, J.H.; Inskip, H.M.; Baird, J. Methods for determining pubertal status in research studies: Literature review and opinions of experts and adolescents. J. Dev. Orig. Health Dis. 2019, 168-187. [CrossRef]

43. Cericato, G.O.; Bittencourt, M.A.V.; Paranhos, L.R. Validity of the assessment method of skeletal maturation by cervical vertebrae: A systematic review and meta-analysis. Dentomaxillofacial Radiol. 2015, 44, 20140270. [CrossRef]

44. Sanders, J.O.; Qiu, X.; Lu, X.; Duren, D.L.; Liu, R.W.; Dang, D.; Menendez, M.E.; Hans, S.D.; Weber, D.R.; Cooperman, D.R. The Uniform Pattern of Growth and Skeletal Maturation during the Human Adolescent Growth Spurt. Sci. Rep. 2017, 7, 1-9. [CrossRef] [PubMed]

45. Ford, P.; Croix, M.D.S.; Lloyd, R.; Meyers, R.W. The Long-Term Athlete Development model: Physiological evidence and application. J. Sports Sci. 2011, 29, 389-402. [CrossRef] [PubMed] 